\title{
ESTILOS DE AFRONTAMIENTO Y DIFERENCIAS DE GÉNERO ANTE LA MUERTE
}

\section{DEATH COPING STYLES AND GENDER DIFFERENCES}

\author{
Marisa Magaña, José Carlos Bermejo Higuera, \\ Marta Villacieros, Rebeca Prieto
}

\author{
Centro de Humanización de la Salud, Centro San Camilo. Tres Cantos, Madrid, España
}

Cómo referenciar este artículo/How to reference this article:

Magaña, M., Bermejo Higuera, J. C., Villacieros, M. y Prieto, R. (2019). Estilos de Afrontamiento y Diferencias de Género ante la Muerte. Revista de Psicoterapia, 30(112), 103-117. https://doi.org/ 10.33898/rdp.v30i112.269

\begin{abstract}
Resumen
El objetivo del estudio fue analizar las relaciones entre los distintos estilos y estrategias de afrontamiento y la capacidad de afrontamiento de la muerte, incluyendo posibles diferencias entre sexos. Además, se analizaron las características psicométricas del COPE abreviado de Carver (1997). Participaron 216 personas asistentes a unas Jornadas de Duelo, el 81\% (175) mujeres, de edad media 49 años (DT=14,59). Se midieron estilos de afrontamiento (cuestionario COPE abreviado de Carver, 1997) y capacidad de afrontamiento de la muerte (AM) (escala de afrontamiento de la muerte; Bugen, 1981). Serealizaron análisis factoriales exploratorios en ambos cuestionarios, análisis de diferencias de medias y correlaciones. Para el cuestionario COPE se ajustó un modelo de nueve factores de primer orden y dos de segundo orden; afrontamiento activo (AA) y evitativo (AE). Las mujeres obtuvieron medias significativamente más altas en $A E(1,65 \mathrm{vs}$ 1,47) y menores en $A M(48,42$ vs 54,05). En mujeres se obtuvo asociación directa entre AM y AA $(r=, 241)$ y entre AA y AE $(r=, 284)$. Ambos sexos asociación directa entre AM y Aceptación ( $r=, 561$ y $r=, 378 ; p<, 001)$. Los hombres, correlación inversa $(r=-, 536 ; p<, 001)$ entre AE y Aceptación. Se valida una estructura de la escala COPE abreviada para población española. Se observa que las mujeres utilizan un mayor repertorio de estrategias de afrontamiento y que, en ellas, no está reñido el uso combinado de ambos estilos de afrontamiento, activo y evitativo, sin embargo, en hombres, el uso del estilo evitativo parece impedir estrategias activas como la aceptación.

Palabras clave: estilos de afrontamiento; estrategias de afrontamiento; afrontamiento de la muerte; diferencias de género; cuestionario COPE
\end{abstract}

\begin{abstract}
The aim of the study was to analyze the relationships between different styles and coping strategies and the death coping competency, including the possibility of gender differences. In addition psychometric characteristics of the abbreviated COPE of Carver (1997) were analysed. They participated 216 people attending a Grief Workshop, 81\% (175) women, age average 49 years $(S D=14,59)$. Coping styles were measured (questionnaire COPE abbreviated; Carver, 1997) and death coping competency (DC) (Coping with Death Scale; Bugen, 1981). Exploratory factor analyses were performed on both questionnaires, mean differences analysis and correlations. A model of nine first order and two second order factore for COPE questionnaire was adjusted; active (Ac) and avoidant (Av) coping. Women obtained significantly higher mean in $A v(1.65 \mathrm{vs} 1.47)$ and minor in DC $(48,42 \mathrm{vs} 54,05)$. Women direct association between DC and Ac ( $r=$, 241) and between Ac and $A v(r=, 284)$ was obtained. Both sexes, direct association between DC and Acceptance $(r=, 561$ and $r=, 378 ; p<, 001)$. The men, inverse correlation ( $r=-$, 536; $p<$, 001) between $A v$ and Acceptance. A COPE scale structure for Spanish population was validated. Shown that women use a greater repertoire of coping strategies and that, in them, combined both styles of coping, active and avoiding use is at odds, however in men using the avoidant style, seems to prevent strategies active as acceptance.
\end{abstract}

Keywords: coping styles; coping strategies; coping with death; gender differences; COPE Questionnaire

\footnotetext{
Fecha de recepción: 23/11/2018. Fecha de aceptación v1: 23/12/2018. Fecha de aceptación v2: 26/01/2019. Correspondencia sobre este artículo:

E-mail: escucha@humanizar.es

Dirección postal: Sector Escultores, 39. 28760 Tres Cantos Madrid, España

(C) 2019 Revista de Psicoterapia
} 
La pérdida de un ser querido es uno de los sucesos vitales estresantes a los que todos nos tendremos que enfrentar en algún momento. Es un fenómeno inevitable, y como tal, constituye un estímulo ansiógeno capaz de afectar a actitudes y comportamientos e incidir en la calidad de la atención que cualquier profesional de la relación de ayuda ha de enfrentar. Por eso se han estudiado causas, correlatos, consecuencias e implicaciones de la ansiedad ante la muerte que pueden resultar útiles en el marco de la atención sanitaria (Neimeyer, 1997).

La capacidad de afrontamiento de la muerte dependerá tanto de las características personales y socioculturales, como de la educación y de la formación recibida. Se ha estudiado respecto a sus diferentes fases (Sadín y Chorot, 2003), respuestas fisiológicas (Selye, 1983) y estrategias utilizadas (Fernández-Abascal, Jiménez y Martín, 2003).

La utilización de distintas estrategias, marcarán diferencias individuales en los estilos de afrontamiento (Galiana, 2015). Lazarus y Folkan (1984) definieron el afrontamiento al estrés como el esfuerzo para manejar las demandas específicas internas y/o externas que son evaluadas como excesivas en relación con los recursos de que dispone la persona, estableciendo dos tipos de afrontamiento: centrado en la emoción (en el que se trata de regular la emoción causada por el distrés) y en el problema (en el que se trata de manejar el problema que lo origina). Rodríguez Marín (1996) distinguió entre formas de afrontamiento activas (o de afrontamiento del problema) y evitativas (que permiten escapar del problema).

Entre las estrategias de afrontamiento más importantes se encuentran la reevaluación positiva, reacción depresiva, negación, planificación, conformismo, desconexión mental, evitación, apoyo social y expresión emocional (FernándezAbascal, et al., 2003).

Los estilos de afrontamiento, definidos por la utilización de un tipo u otro de estrategias, han sido estudiados en diferentes ámbitos como el laboral (Martínez, 2015), el económico (Riquelme, Rodríguez y Buendía, 1993) y el académico (Morales y García, 2017). Ante procesos de muerte y duelo podemos también hablar de estilos de afrontamiento activo y evitativo (Carver, 1989, 1997), centrado en los problemas, en las emociones o en la búsqueda de la trascendencia (López, Crespo, Arinero, Gómez y Francisco, 2003).

Cada vez más estudios destacan la importancia que los estilos de afrontamiento en la elaboración del duelo (Elvira de la Morena y Cruzado, 2013; Wartella, Auerbach y Ward, 2009). De hecho, parece que las personas que tardan más tiempo en pedir ayuda específica para elaborar el duelo (e.g., afrontamiento evitativo) frente a las personas que acuden rápidamente a pedir ayuda (e.g., afrontamiento activo), acaban necesitando una mayor atención profesional para su problema y viviendo un duelo complicado (Villacieros, Magaña, Bermejo, Carabias y Serrano, 2012).

Una de las variables que influye en los estilo de afrontamiento ante la muerte y procesos de duelo, es el género, construcción social derivada de los diferentes 
procesos de socialización. La investigación muestra, por un lado, que los cerebros de hombres y mujeres están construidos de manera diferente, percibiendo y procesando la información de manera diferencial y, por otro lado, que hombres y mujeres reciben un trato diferencial que marca una distinción entre pensamientos, conductas, creencias, por lo que también responden al duelo condicionados por las expectativas puestas sobre ellos. Es frecuente que los hombres hagan su duelo volviendo tan pronto como pueden a su actividad normal, evitando pensar sobre ello, y con más facilidad expresando ira que tristeza. No expresan el duelo de manera afectiva y algunos autores consideran que responden al duelo de manera no saludable (Corr, Nabe y Corr, 2000). Por el contrario, con frecuencia las mujeres son más emocionales, más expresivas sobre la pérdida, buscan ayuda y apoyo en otros lo que es necesario en el duelo y sienten más haciendo a otros participes de sus sentimientos más íntimos (Staudacher, 1991).

Por eso, en un inicio se nombraron estas diferencias de género como duelo masculino y femenino (Corr et al., 2000; Nolen-Hoeksema y Larson, 1999; Stinson y Lasker, 1992). Así, existen estudios que concluyen que las mujeres utilizan en mayor medida la estrategia de búsqueda de apoyo, mientras que los hombres tienden más a fijarse en lo positivo, utilizando estrategias de distracción (Martín, Lucas y Pulido, 2011), o que los hombres puntúan más alto en estrategias como reinterpretación positiva y planificación y las mujeres en estrategias de apoyo social (Cabanach, Fariña, Freire, González y Ferradás, 2013). Otros estudios encuentran puntuaciones más elevadas en mujeres en estrategias de apoyo emocional y religión, y en hombres estilos de afrontamiento evitativo y humor (Morán, Landero y González, 2013); otros no encuentran diferencias significativas en estrategias utilizadas en función del sexo (Bermejo, Magaña, Villacieros, Carabias y Serrano, 2012).

Martin y Doka $(1996,2000)$ proponen un modelo de dos patrones de afrontamiento mayoritarios. Definen el duelo instrumental y duelo intuitivo, en vez de masculino y femenino, como polos de un continuo desde lo más resolutivocognitivo hasta lo más emotivo-expresivo, reconociendo la variedad de respuestas en cualquiera de los dos sexos. Así, tanto hombres como mujeres pueden moverse en los distintos niveles de los dos patrones, incluso situarse en el punto medio haciendo uso de ambos patrones.

Ninguno de los patrones es más o menos eficaz respecto al duelo, sino que ambos pueden ser adaptativos (Bonanno, Keltner, Holen y Horowitz, 1995; Martin y Doka, 2000; Osterweis, Solomon y Green, 1984). De hecho, ambos perfiles pueden beneficiarse de este conocimiento; mientras que las estrategias puestas en marcha inicialmente reflejan las fortalezas naturales de cada uno, ser confrontados les permitirá un afrontamiento más holístico.

Para evaluar tanto estilos como estrategias de afrontamiento ante procesos de duelo, uno de los cuestionarios más utilizados en castellano es el COPE Abreviado de Carver (1997), versión reducida del original (Carver, 1989), que cuenta con 
diversos estudios pero con consenso limitado en cuanto a su dimensionalidad. Inicialmente Carver (1997), estableció 9 factores de primer orden y dos de segundo orden, estilo activo y evitativo. Sin embargo, Perczek, Carver, Price y PozoKaderman (2014) obtuvieron 12 factores subyacentes. Por su parte, López et al. (2003), en cuidadores de mayores dependientes y después de excluir tres ítems, encontraron 12 factores de primer orden y tres de segundo orden; afrontamiento centrado en los problemas, en las emociones y búsqueda de trascendencia.

Por otro lado, para evaluar la capacidad de afrontamiento ante la muerte, un cuestionario ampliamente utilizado en nuestro contexto es el Coping with Death Scale (Bugen, 1980). En su versión traducida al castellano existen diferentes estudios. Uno de ellos centrado en universitarios (Schmidt, 2007) y otro en una muestra de profesionales de cuidados paliativos (Galiana, Oliver, Sansó, Pades y Benito, 2017). Ambos apoyan la estructura unidimensional propuesta por Bugen (1980).

El objetivo del presente trabajo fue estudiar las relaciones entre los distintos estilos y estrategias de afrontamiento y la capacidad de afrontamiento de la muerte, incluyendo el análisis pormenorizado de las diferencias entre sexos. Con el fin de confirmar la validez y fiabilidad del COPE abreviado de Carver (1997), además, se analizó su dimensionalidad en esta muestra.

\section{Método}

\section{Participantes}

El cuestionario de investigación fue contestado por 216 personas asistentes a las Jornadas de Duelo realizadas en un centro sociosanitario de la Comunidad de Madrid. Del total, 175 (81\%) eran mujeres y $41(19 \%)$ hombres con una media de edad de 49 años (DT=14.59, mín.=18, máx.=81).

\section{Instrumento}

El cuestionario incluía variables sociodemográficas (sexo, edad, estado civil y estudios), sobre duelos por fallecimiento (experiencias cercanas, justificación y pérdida de ser querido), experiencias de enfermedad y accidentes graves, religión y práctica de la misma.

Tras ellas se les pedía a los participantes que pensaran en una pérdida significativa (intensa, difícil o costosa) que hayan tenido que vivir seleccionando una de ellas (divorcio, separación, enfermedad, trabajo o rol migración, fallecimiento incluyendo abortos, desaparición, otra) para después responder a las preguntas del cuestionario en referencia a esta experiencia;

- Estilos de afrontamiento: cuestionario COPE abreviado de Carver(1997) de 28 ítems, con formato de respuesta tipo Likert desde 1 ( No, en absoluto), hasta 4 (Sí, mucho). Evalúa los estilos de afrontamiento activo (búsqueda de apoyo social emocional e instrumental, planificación y afrontamiento 
activo, desahogo, reinterpretación positiva, humor, aceptación, religión y autoculpabilización; y evitativo: autodistracción, negación, consumo de alcohol y drogas y desconexión conductual.

- Capacidad de afrontamiento de la muerte: escala de afrontamiento de la muerte de Bugen (1980). Para no alargar el cuestionario se seleccionaron 10 de los 30 ítems de la escala en castellano publicada por Galiana et al. (2017). Cada ítem se valora del 1 (totalmente en desacuerdo) al 7 (totalmente de acuerdo). En la suma total, a mayor puntuación, mayor capacidad de afrontamiento de la muerte.

\section{Procedimiento}

El cuestionario se entregó a la entrada de las Jornadas de Duelo del centro, en la mesa de inscripciones. Se pidió a los asistentes que lo rellenasen y entregasen en unas urnas destinadas a ello.

\section{Análisis de datos}

Se realizaron análisis factoriales exploratorios (AFE) de primer y segundo orden con el Cuestionario COPE para valorar la dimensionalidad mediante el programa R. Previo análisis, se comprobaron el supuesto de normalidad multivariante a través del Test de Mardia, y la posibilidad de factorizar la matriz muestral a través de la Prueba de Bartlett y el índice KMO. El método de análisis fue mínimos cuadrados no ponderados (ULS) y rotación Oblimin. Para la elección del modelo se tuvieron en cuenta gráfico de sedimentación, la prueba de análisis paralelos y los siguientes índices de ajuste (Abad, Olea, Ponsoda y García, 2011): Chi cuadrado/ grados de libertad $\left(X^{2} / \mathrm{gl}<3\right.$ puntos se considera un ajuste aceptable), el Error Cuadrático Medio de Aproximación (RMSEA $<.08$ indica ajuste aceptable), el Error Cuadrático Medio Estandarizado (SRMR $<.08$ indica buen ajuste de los datos) (Hu y Bentler, 1998), el Índice de Tucker-Lewis (TLI $>.90$ indica ajuste aceptable). Se comprobó la fiabilidad mediante el índice de consistencia interna alfa de Cronbach.

El análisis de diferencias de medias mediante el programa SPSS v.22 para aquellas variables con respuesta dicotómica se realizó mediante la Prueba T de Student para muestras independientes o Mann-Whitney, dependiendo del cumplimiento del supuesto de normalidad (que se comprobó mediante la prueba de Kolmogorov-Smirnov). Las diferencias de medias con respuesta de más de dos categorías a través de la prueba ANOVA de un factor ó Kruskal Wallis, dependiendo de si las escalas cumplían normalidad. Los análisis post hoc se utilizó la prueba de Tukey, ya que solo afectó a una escala que cumplió tanto el supuesto de normalidad como el de homocedasticidad (comprobado mediante la prueba de Levene). Se calcularon las correlaciones de Pearson entre las variables cuantitativas. 


\section{Resultados}

\section{Descripción de la muestra}

Edad media de 49 años (DT=15 y Mna=52) (Tabla 1), la mitad de ellos casados o en pareja y con estudios mayoritariamente sociosanitarios $(62 \%)$. Dos terceras partes profesaban alguna religión y casi la mitad se consideraban practicantes.

Más del $80 \%$ refiere no haber sufrido enfermedad o accidente importante, pero sí enfermos graves en la familia (70.8\%). El 91\% han sufrido la pérdida de un ser querido o familiar próximo. En cuanto a la pérdida significativa sobre la que responden al cuestionario, de los 216 cuestionarios, 202 seleccionan una opción. De los 14 que no lo hicieron, se comprobó que 9 de ellos habían contestado Sí a la pregunta ¿Tiene experiencia cercana de duelos por fallecimiento?, y 11 a la pregunta ¿Ha sufrido la pérdida de un familiar próximo o ser querido? Solamente un participante había contestado "No" a ambas preguntas, por lo que se eliminó ese cuestionario. Entre los 202 que seleccionaron una opción, la experiencia mayoritaria fue el fallecimiento (39.4\%). Sumando las categorías fallecimiento, desaparición de ser querido y separación/divorcio, se obtuvieron 150 personas (casi $70 \%$ de la muestra), que contesta al cuestionario referido a la ausencia de un ser querido.

Tabla 1. Características de la muestra.

\begin{tabular}{|c|c|c|c|}
\hline \multicolumn{2}{|c|}{ Variables y opciones de respuesta } & \multirow{2}{*}{$\frac{\mathbf{N}}{108}$} & \multirow{2}{*}{$\frac{\%}{50}$} \\
\hline Estado Civil & Casado/a o en pareja & & \\
\hline & Soltero/a & 59 & 27.3 \\
\hline & Viudo/a & 15 & 6.9 \\
\hline & Separado/a o divorciado/a & 20 & 9.3 \\
\hline & Religioso/a & 10 & 4.6 \\
\hline \multirow[t]{8}{*}{ Estudios } & Enfermería & 18 & 8.3 \\
\hline & Medicina & 6 & 2.8 \\
\hline & Aux.Enfermería & 22 & 10.2 \\
\hline & Psicología & 30 & 13.9 \\
\hline & Trabajo Social & 14 & 6.5 \\
\hline & Oros Sanitarios & 20 & 9.3 \\
\hline & Otros Sociales & 24 & 11.1 \\
\hline & Otros & 78 & 36.11 \\
\hline $\begin{array}{l}\text { ¿Tiene experiencia cercana de } \\
\text { duelos por fallecimiento? }\end{array}$ & $\begin{array}{l}\text { Sí } \\
\text { No }\end{array}$ & $\begin{array}{r}187 \\
25\end{array}$ & $\begin{array}{l}86.6 \\
11.6\end{array}$ \\
\hline Experiencia en duelos debido a..., & $\begin{array}{l}\text { Rol (profesión, cuidador...) } \\
\text { Intereses o experiencias personales } \\
\text { Ambos }\end{array}$ & $\begin{array}{l}45 \\
91 \\
63\end{array}$ & $\begin{array}{l}20.8 \\
42.1 \\
31.7\end{array}$ \\
\hline Ud. ¿Ha sufrido o sufre alguna & Sí & 35 & 16.2 \\
\hline enfermedad grave? & No & 176 & 81.5 \\
\hline
\end{tabular}




\begin{tabular}{|c|c|c|c|}
\hline \multirow{2}{*}{$\begin{array}{l}\text { Ud. ¿Ha sufrido algún accidente } \\
\text { importante? }\end{array}$} & Sí & 29 & 13.4 \\
\hline & No & 181 & 83.8 \\
\hline \multirow{2}{*}{$\begin{array}{l}\text { En su familia, ¿hay o ha habido } \\
\text { algún enfermo grave? }\end{array}$} & Sí & 153 & 70.8 \\
\hline & No & 60 & 27.8 \\
\hline \multirow{2}{*}{$\begin{array}{l}\text { ¿Ha sufrido la pérdida de un familiar } \\
\text { próximo o ser querido? }\end{array}$} & Sí & 197 & 91.2 \\
\hline & No & 16 & 7.4 \\
\hline \multirow[t]{2}{*}{ ¿Profesa alguna religión? } & Sí & 146 & 67.6 \\
\hline & No & 66 & 30.6 \\
\hline \multirow[t]{2}{*}{ ¿Se considera practicante? } & Sí & 104 & 48.1 \\
\hline & No & 101 & 46.8 \\
\hline \multirow{7}{*}{$\begin{array}{l}\text { Pérdida significativa según la que } \\
\text { se responde al cuestionario }\end{array}$} & Divorcio/Separación & 33 & 15.3 \\
\hline & Fallecimiento (incluye abortos) & 85 & 39.4 \\
\hline & Desaparición de ser querido & 32 & 14.8 \\
\hline & Enfermedad (incluye discapacidades) & 20 & 9.3 \\
\hline & Trabajo o rol & 17 & 7.9 \\
\hline & Migración & 9 & 4.2 \\
\hline & Otra & 6 & 2.8 \\
\hline
\end{tabular}

\section{Análisis factorial exploratorio de los cuestionarios (COPE y Bugen)}

Dada la variabilidad publicada en cuanto a su estructura interna el cuestionario COPE se puso a prueba mediante AFE. En la tabla 2 se presentan los indicadores de ajuste de los modelos obtenidos de primer y segundo orden. En ambos modelos fueron adecuados (Tabla 2). Además, los pesos obtenidos tras la rotación resultaron significativos $(\mathrm{p}<.001)$ y sustantivos (mayores a .4) excepto para el ítem 25 (.373) y excepto para la escala de $\mathrm{d}$ consumo de alcohol y drogas (.357).

Tabla 2. Análisis Factorial Exploratorio e índices de ajuste para modelos de $1^{\circ}$ y $2^{\circ}$ orden

\begin{tabular}{|c|c|c|c|c|c|c|c|c|c|c|c|c|}
\hline ORDEN & MODELO & $\chi^{2}(p)$ & $g \mid$ & $\chi^{2} / g \mid$ & RMSEA & $\Delta \chi^{2}$ & $\Delta \mathrm{gl}$ & $\Delta \chi^{2}(p)$ & $\%$ VAR & $\%$ RES & SRMR & TLI \\
\hline 10 & 9 Factores & $\begin{array}{l}104.34 \\
(.990)\end{array}$ & 144 & .72 & .06 & 25.75 & 18 & .106 & 56 & 8 & .03 & .90 \\
\hline $2^{0}$ & 2 Factores & $\begin{array}{l}11.75 \\
(.900)\end{array}$ & 19 & .62 & .09 & 6.01 & 7 & .539 & 34 & 22 & .03 & .93 \\
\hline
\end{tabular}

El modelo final se presenta en la tabla 3 junto a los índices de fiabilidad de cada escala. Las diferencias respecto a la dimensionalidad de la versión de Carver (1997) consistieron en, por un lado, la reducción de Estilo de afrontamiento activo (AA) de 9 a 6 factores, y por otro, la reducción de Estilo de afrontamiento evitativo (AE) de 4 a 3 factores. 
Tabla 3. Estructura factorial COPE para este estudio. Ítems e índices de fiabilidad de cada escala

\begin{tabular}{|c|c|c|}
\hline SEGUNDO ORDEN & PRIMER ORDEN & ÍTEMS \\
\hline \multirow{18}{*}{$\begin{array}{l}\text { Estilo de } \\
\text { Afrontamiento } \\
\text { Activo (AA) } \\
(\alpha=69)\end{array}$} & \multirow{5}{*}{$\begin{array}{l}\text { Búsqueda de Apoyo } \\
\text { Social (emocional e } \\
\text { instrumental) } \\
\quad(\alpha=84)\end{array}$} & 5. He estado consiguiendo apoyo emocional de los demás. \\
\hline & & 10. He estado recibiendo ayuda y consejo de otras personas. \\
\hline & & 15. He estado consiguiendo consuelo y comprensión de alguien. \\
\hline & & 21. He estado expresando mis sentimientos negativos. \\
\hline & & $\begin{array}{l}\text { 23. He estado intentando conseguir consejo } 0 \text { ayuda de otros } \\
\text { acerca de qué hacer. }\end{array}$ \\
\hline & \multirow{5}{*}{$\begin{array}{l}\text { Planificación (y } \\
\text { desahogo) } \\
(\alpha=.75)\end{array}$} & $\begin{array}{l}\text { 2. He estado concentrando mis esfuerzos en hacer algo acerca } \\
\text { de la situación en la que estoy. }\end{array}$ \\
\hline & & 7. He estado dando pasos para intentar mejorar la situación. \\
\hline & & $\begin{array}{l}\text { 9. He estado diciendo cosas para dejar salir mis sentimientos } \\
\text { desagradables. }\end{array}$ \\
\hline & & $\begin{array}{l}\text { 14. He estado intentando dar con alguna estrategia acerca de } \\
\text { qué hacer. }\end{array}$ \\
\hline & & 25. He estado pensando mucho acerca de los pasos a tomar. \\
\hline & \multirow{2}{*}{ Humor $(\alpha=.65)$} & 18. He estado haciendo bromas sobre ello. \\
\hline & & 28. He estado burlándome de la situación. \\
\hline & \multirow[t]{2}{*}{ Religión $(\alpha=.87)$} & $\begin{array}{l}\text { 22. He estado intentando encontrar consuelo en mi religión o } \\
\text { creencias espirituales. }\end{array}$ \\
\hline & & 27. He estado rezando o meditando. \\
\hline & \multirow[t]{2}{*}{ Aceptación $(\alpha=.66)$} & $\begin{array}{l}\text { 20. He estado aceptando la realidad del hecho de que ha } \\
\text { ocurrido. }\end{array}$ \\
\hline & & 24. He estado aprendiendo a vivir con ello. \\
\hline & \multirow{2}{*}{$\begin{array}{l}\text { Reinterpretación } \\
\text { positiva }(\alpha=.64)\end{array}$} & $\begin{array}{l}\text { 12. He estado intentando verlo bajo otro aspecto que lo haga } \\
\text { parecer más positivo. }\end{array}$ \\
\hline & & 17. He estado buscando algo bueno en lo que está pasando. \\
\hline \multirow{10}{*}{$\begin{array}{l}\text { Estilo de } \\
\text { Afrontamiento } \\
\text { Evitativo (AE) } \\
\qquad(\alpha=.57)\end{array}$} & \multirow{6}{*}{$\begin{array}{l}\text { Estrategias } \\
\text { (negación, } \\
\text { autodistracción, } \\
\text { desconexión } \\
\text { conductual) }(\alpha=.74)\end{array}$} & $\begin{array}{l}\text { 1. Me he estado volcando en el trabajo y otras actividades para } \\
\text { apartar mi mente de cosas desagradables. }\end{array}$ \\
\hline & & 3. Me he estado diciendo a mí mismo "esto no es real". \\
\hline & & 6. He estado dejando de intentar hacerle frente. \\
\hline & & 8. Me he estado negando a creer que esto ha sucedido. \\
\hline & & 16. He estado dejando de intentar afrontarlo. \\
\hline & & $\begin{array}{l}\text { 19. He estado haciendo cosas para pensar menos en ello, como } \\
\text { ir al cine, ver la televisión, leer, soñar despierto, dormir o ir de } \\
\text { compras. }\end{array}$ \\
\hline & \multirow{2}{*}{ Consumo $(\alpha=.90)$} & $\begin{array}{l}\text { 4. He estado tomando alcohol u otras drogas para sentirme } \\
\text { mejor. }\end{array}$ \\
\hline & & $\begin{array}{l}\text { 11. He estado consumiendo alcohol u otras drogas para } \\
\text { ayudarme a superarlo. }\end{array}$ \\
\hline & \multirow{2}{*}{$\begin{array}{l}\text { Autoculpabilización } \\
\qquad(\alpha=.81)\end{array}$} & 13. Me he estado criticando a mí mismo. \\
\hline & & $\begin{array}{l}\text { 26. Me he estado culpando a mí mismo por cosas que } \\
\text { sucedieron. }\end{array}$ \\
\hline
\end{tabular}


Respecto a la escala Bugen de Afrontamiento de la Muerte (AM), el AFE indicó una estructura unidimensional con índices de ajuste aceptables y el alfa de Cronbach obtenido fue de .90 .

Diferencias en los estilos de afrontamiento y capacidad de afrontamiento de la muerte; sexo, religión, práctica, estudios y tipo de pérdida

En la Tabla 4 se presentan las variables dicotómicas para las que se hallaron diferencias significativas en las medias de estas tres variables. La variable sexo arrojó diferencias en AE y AM. Las mujeres obtienen una media más alta en $\mathrm{AE}$ (1.65 vs. 1.47) y menor en AM (48.42 vs. 54.05). No se encontraron diferencias entre hombres y mujeres en las subescalas de ninguno de los dos estilos de afrontamiento.

Para la variable religión se encontraron diferencias en ambos estilos de afrontamiento. Los que dicen profesar alguna religión obtienen una media más alta (2.37 vs. 2.20) en AA y más baja en AE (1.55 vs. 1.72). El hecho de ser practicante también influye en las escalas AA y AM, de manera que la media de los que practican es más alta (2.43 vs. 2.20) y muestran mayor capacidad AM (51.42 vs. 48).

Tabla 4. Diferencias de medias entre las categorías formadas por las variables sexo, religión y práctica para las variables principales del estudio.

\begin{tabular}{|c|c|c|c|c|c|c|c|}
\hline & & \multicolumn{2}{|c|}{ Sexo } & \multicolumn{2}{|c|}{ Religión } & \multicolumn{2}{|c|}{ Practicante } \\
\hline & & Hombre & Mujer & Sí & No & Sí & No \\
\hline & $\mathbf{N}$ & 41 & 171 & 143 & 65 & 102 & 99 \\
\hline $\begin{array}{c}\text { Afrontamiento } \\
\text { activo }\end{array}$ & $\begin{array}{c}\text { Media } \\
D T \\
p\end{array}$ & & & $\begin{array}{r}2.37 \\
.48 \\
.0\end{array}$ & $\begin{array}{l}2.2 \\
.54 \\
3 \\
\end{array}$ & $\begin{array}{l}2.43 \\
.5 \\
\quad .0\end{array}$ & $\begin{array}{l}2.2 \\
.49 \\
2 \\
\end{array}$ \\
\hline \multirow[t]{2}{*}{$\begin{array}{c}\text { Afrontamiento } \\
\text { evitativo }\end{array}$} & $\begin{array}{c}\text { Media } \\
D T \\
p\end{array}$ & $\begin{array}{ll}1.47 & \\
.46 & \\
& .0 \\
\end{array}$ & $\begin{array}{r}1.65 \\
.56 \\
\end{array}$ & $\begin{array}{c}1.55 \\
.53 \\
.0\end{array}$ & $\begin{array}{l}1.72 \\
.53 \\
4 \\
\end{array}$ & & \\
\hline & $\mathbf{N}$ & 39 & 147 & & & 90 & 87 \\
\hline $\begin{array}{l}\text { Afrontamiento } \\
\text { de la muerte }\end{array}$ & $\begin{array}{c}\text { Media } \\
D T \\
p\end{array}$ & $\begin{array}{c}54.05 \\
7.97 \\
.0\end{array}$ & $\begin{array}{l}48.42 \\
13.14 \\
17\end{array}$ & & & $\begin{array}{r}51.42 \\
11.86 \\
.0\end{array}$ & $\begin{array}{l}48 \\
12.99 \\
48\end{array}$ \\
\hline
\end{tabular}

En la variable estudios solo se encontraron diferencias significativas entre las categorías de la escala AA y AM. En tipos de pérdida en la escala AA, por lo que se procedió a los análisis post hoc entre las categorías de estas dimensiones (Tabla 5).

Dentro de la variable estudios, las categorías que obtuvieron diferencias estadísticamente significativas en sus medias de AA fueron los tres grupos de mayor media en AA, psicología $(\mathrm{p}=.003)$, otros sociales $(\mathrm{p}=.014)$ y otros $(\mathrm{p}=.047)$, (medias de $2.50,2.50$ y 2.34 respectivamente) con respecto al grupo de menor media en AA auxiliar de enfermería (1.90). 
Tabla 5. Diferencias de medias entre las categorías formadas por las variables estudios y tipos de pérdida.

\begin{tabular}{|c|c|c|c|c|c|c|c|c|c|}
\hline Estudios & & Medicina & Enfermería & Psicología & $\begin{array}{l}\text { Trabajo } \\
\text { Social }\end{array}$ & $\begin{array}{l}\text { Auxiliar de } \\
\text { Enfermería }\end{array}$ & $\begin{array}{c}\text { Otros } \\
\text { sociales }\end{array}$ & $\begin{array}{c}\text { Otros } \\
\text { sanitarios }\end{array}$ & Otros \\
\hline Afrontamiento & $\begin{array}{c}N \\
\text { Media } \\
D T \\
p\end{array}$ & $\begin{array}{c}6 \\
2.67 \\
.42\end{array}$ & $\begin{array}{c}18 \\
2.29 \\
.43\end{array}$ & $\begin{array}{l}30 \\
2.5 \\
.48\end{array}$ & $\begin{array}{c}14 \\
2.39 \\
.35 \\
<.001 \\
\end{array}$ & $\begin{array}{l}21 \\
1.9 \\
.38\end{array}$ & $\begin{array}{l}23 \\
2.5 \\
.37\end{array}$ & $\begin{array}{c}18 \\
2.12 \\
.6\end{array}$ & $\begin{array}{c}78 \\
2.34 \\
.53\end{array}$ \\
\hline $\begin{array}{l}\text { Afrontamiento } \\
\text { de la muerte }\end{array}$ & $\begin{array}{c}N \\
\text { Media } \\
D T \\
p\end{array}$ & $\begin{array}{c}6 \\
50.17 \\
13.04\end{array}$ & $\begin{array}{c}18 \\
56.05 \\
11.21\end{array}$ & $\begin{array}{c}28 \\
52.29 \\
10.01\end{array}$ & $\begin{array}{c}12 \\
49.83 \\
9.85 \\
.030\end{array}$ & $\begin{array}{c}20 \\
43.45 \\
12.86\end{array}$ & $\begin{array}{c}21 \\
53.19 \\
8.81\end{array}$ & $\begin{array}{c}14 \\
41.21 \\
17.82\end{array}$ & $\begin{array}{c}65 \\
49.54 \\
11.94\end{array}$ \\
\hline Tipos de pérdida & & $\begin{array}{c}\text { Trabajo } \\
\text { rol }\end{array}$ & $\begin{array}{c}\text { Divorcio } \\
\text { Separación }\end{array}$ & $\begin{array}{l}\text { Desapa- } \\
\text { rición }\end{array}$ & $\begin{array}{l}\text { Falleci- } \\
\text { miento }\end{array}$ & $\begin{array}{l}\text { Enferme- } \\
\text { dad }\end{array}$ & Migración & Otra & \\
\hline $\begin{array}{c}\text { Afrontamiento } \\
\text { activo }\end{array}$ & $\begin{array}{c}N \\
\text { Media } \\
D T \\
p\end{array}$ & $\begin{array}{c}17 \\
2.57 \\
.6\end{array}$ & $\begin{array}{c}33 \\
2.49 \\
.41\end{array}$ & $\begin{array}{c}32 \\
2.39 \\
.48 \\
<.001\end{array}$ & $\begin{array}{c}84 \\
2.27 \\
.45\end{array}$ & $\begin{array}{c}20 \\
2.08 \\
.55\end{array}$ & $\begin{array}{c}9 \\
1.8 \\
.57\end{array}$ & $\begin{array}{c}5 \\
2.6 \\
.3\end{array}$ & \\
\hline
\end{tabular}

En AM, los participantes con estudios de enfermería obtuvieron una media significativamente $(\mathrm{p}<.05)$ mayor $(56.05)$ que los tres grupos con medias inferiores; otros sanitarios (41.21; $\mathrm{p}=.014)$, auxiliares de enfermería (43.45; $\mathrm{p}=.003)$ y otros (49.54; $\mathrm{p}=.047)$. Y, de nuevo, los auxiliares de enfermería obtuvieron medias estadísticamente $(\mathrm{p}<.05)$ menores $(43.45)$ que otros cuatro grupos de estudios; los tres con mayores medias y mayores $\mathrm{N}$; enfermería, otros sociales $(53.19 ; \mathrm{p}=.007)$, psicología (52.29; $\mathrm{p}=.013)$ y otros $(49.54 ; \mathrm{p}=.037)$.

Dentro de tipos de pérdida, la media en afrontamiento activo en el grupo enfermedad (2.08) fue significativamente $(\mathrm{p}=.036)$ menor que en divorcio/separación (2.49) y que en trabajo/rol (2.49; $\mathrm{p}=.003)$ y la media en AA en migración(1.80), fue significativamente menor que en divorcio/separación $(\mathrm{p}=.037)$, trabajo/rol $(\mathrm{p}=.003)$ y desaparición de un ser querido (2.39; $\mathrm{p}=.023)$.

Para AE, las medias de los distintos tipos de estudios oscilaron entre 1,3 $(\mathrm{DT}=.26)$ en medicina y $1.72(\mathrm{DT}=.59)$ en otros sociales. Para los grupos formados por tipos de pérdidas, las medias de EA oscilaron entre 1.40 (DT=.37) de enfermedad y $1,89(\mathrm{DT}=.96)$ de otras.

\section{Análisis de correlaciones entre estilos de afrontamiento y capacidad de afrontamiento de la muerte en ambos sexos}

Respecto a la asociación entre la capacidad de afrontamiento ante la muerte y los dos estilos principales de afrontamiento (Tabla 6, mitad superior), se observa que en ambos sexos existe correlación inversa entre $\mathrm{AM}$ y estilo $\mathrm{AE}$, siendo esta asociación más intensa en el caso de los hombres $(r=-.396$ vs $r=-.174)$. En el caso de la mujeres, además existe asociación directa entre AM y AA $(r=.241)$ y entre 
ambos estilos de afrontamiento $(\mathrm{r}=.284)$.

Tabla 6. Correlaciones de Pearson entre las variables principales del estudio según sexos.

\begin{tabular}{|c|c|c|c|c|c|c|}
\hline \multirow{2}{*}{$\begin{array}{l}\text { Correlaciones } \\
\text { de Pearson }\end{array}$} & \multicolumn{3}{|c|}{ Hombres } & \multicolumn{3}{|c|}{ Mujeres } \\
\hline & AA & $\mathrm{AE}$ & AM & $A A$ & $\mathrm{AE}$ & AM \\
\hline $\begin{array}{l}\text { Afrontamiento Activo (AA) } \\
\text { Afrontamiento Evitativo (AE) } \\
\text { Afrontamiento de la Muerte (AM) }\end{array}$ & $\begin{array}{c}-.102 \\
.278\end{array}$ & $-.396^{\star}$ & & & $.284^{\star \star}$ & $\begin{array}{l}.241^{\star \star} \\
-.174^{\star}\end{array}$ \\
\hline $\begin{array}{l}\text { AA: Búsqueda de apoyo social } \\
\text { AA: Planificación } \\
\text { AA: Humor } \\
\text { AA: Religión } \\
\text { AA: Aceptación } \\
\text { AA: Reinterpretación positiva }\end{array}$ & $\begin{array}{l}.522^{\star *} \\
.615^{\star *} \\
.398^{\star *} \\
.705^{\star *} \\
.613^{\star *} \\
.703^{\star *}\end{array}$ & $\begin{array}{c}.128 \\
.29 \\
.168 \\
-.128 \\
-.536^{\star \star} \\
-0.189 \\
\end{array}$ & $\begin{array}{l}-.103 \\
-.064 \\
.249 \\
.218 \\
.561^{\star \star} \\
.143 \\
\end{array}$ & $\begin{array}{l}.657^{\star *} \\
.770^{\star *} \\
.418^{\star *} \\
.651^{\star *} \\
.599^{\star *} \\
.741^{\star *}\end{array}$ & $\begin{array}{l}.282^{\star \star} \\
.364^{\star \star} \\
.15 \\
.021 \\
-.059 \\
.240^{\star \star} \\
\end{array}$ & $\begin{array}{l}.055 \\
.136 \\
.176^{\star} \\
.179^{\star} \\
.378^{\star \star} \\
.214^{\star \star}\end{array}$ \\
\hline $\begin{array}{l}\text { AE: Estrategias } \\
\text { AE: Consumo } \\
\text { AE: Autoculpabilización }\end{array}$ & $\begin{array}{l}-.125 \\
-.006 \\
-.1\end{array}$ & $\begin{array}{l}.885^{\star \star} \\
.552^{\star \star} \\
.858^{\star \star}\end{array}$ & $\begin{array}{l}-.505^{\star \star} \\
.021 \\
-.406^{\star \star}\end{array}$ & $\begin{array}{l}.213^{* *} \\
-.044 \\
.239^{* *}\end{array}$ & $\begin{array}{l}.789^{\star \star} \\
.529^{\star \star} \\
.843^{\star \star}\end{array}$ & $\begin{array}{l}-.299^{\star \star} \\
-0.102 \\
-0.099\end{array}$ \\
\hline
\end{tabular}

${ }^{* *}$. La correlación es significativa al nivel .01 (bilateral).

*. La correlación es significante al nivel .05 (bilateral).

Respecto a la asociación entre AM y estrategias (Tabla 6, mitad inferior), se observan diferencias y similitudes entre hombres y mujeres. Mientras que en mujeres existe asociación directa entre AM y las estrategias de AA: humor $(\mathrm{r}=.176$; $\mathrm{p}<.05)$, religión $(\mathrm{r}=.179 ; \mathrm{p}<.05)$, aceptación $(\mathrm{r}=.378 ; \mathrm{p}<.01)$ y reinterpretación positiva $(\mathrm{r}=.214 ; \mathrm{p}<.01)$, en hombres AM solamente se asocia positivamente con la estrategia AA: Aceptación $(\mathrm{r}=.561 ; \mathrm{p}<.01)$. Por otro lado, AM se asocia inversamente con AE: Estrategias en ambos sexos $(\mathrm{r}=-.505 \mathrm{y} \mathrm{r}=-.299 ; \mathrm{p}<.05)$, mientras que en hombres se asocia, además, con AE: Autoculpabilización ( $\mathrm{r}=-.406 ; \mathrm{p}<.01)$.

Respecto estilos y estrategias, además de las asociaciones esperadas (estilo EA con estrategias de EA y estilo AA con estrategias de AA), en el grupo de mujeres se observa asociación directa del estilo AE con tres subescalas de AA (búsqueda de apoyo social, planificación y reinterpretación positiva) $(\mathrm{r}=.282, \mathrm{r}=.364$ y r=.240 respectivamente; $\mathrm{p}<.01$ ) y en el grupo de hombres asociación inversa con la escala de AA: Aceptación $(\mathrm{r}=-.536 ; \mathrm{p}<.01)$.

Por último, destacar que la subescala AA: Aceptación es la que mayor asociación obtiene con AM, tanto en hombres como en mujeres $(\mathrm{r}=.561 \mathrm{y} \mathrm{r}=.378$; $\mathrm{p}<.01)$.

\section{Discusión y Conclusiones}

El objetivo de este estudio fue estudiar la relación entre los distintos estilos y estrategias de afrontamiento y la capacidad de afrontamiento de la muerte, profundizando en las diferencias que pudieran existir entre hombres y mujeres. Para poder hacerlo se procedió primero al estudio pormenorizado de las características 
psicométricas de los cuestionarios utilizados.

Respecto a la estructura bidimensional del COPE, en este estudio se confirman los dos constructos principales de la teoría de Carver (1989; 1997), las dimensiones de estilo de afrontamiento activo y evitativo, con una sola variación dentro de las subescalas que engloban; la estrategia autoculpabilización se traslada del estilo de afrontamiento activo al evitativo. Es común que, ante situaciones de pérdida o muerte, la persona se culpabilice a sí misma o se haga reproches en relación al fallecido, por lo que hizo, por lo que dejó de hacer o porque pudo haberlo evitado. Aunque durante las primeras fases del duelo el sentimiento de culpa puede cumplir una función adaptativa, según Magaña y Bermejo (2013), en la medida en la que una culpa infundada se mantiene o se cronifica, se habla de evitación, de dificultad para afrontar la muerte o para entrar en el proceso de duelo. Por ello encontramos mayor sentido en que la subescala de autoculpabilización se incluya en el estilo de afrontamiento evitativo. El resto de cambios respecto al COPE original se habían obtenido ya en otros estudios (Hair, Anderson, Tatham y Black, 1999; Morán et al., 2010; Perczek et al., 2014), por lo que podemos confirmar la validez y fiabilidad del instrumento utilizado, obteniendo además una estructura más parsimoniosa.

Respecto a la asociación de estilos y estrategias con capacidad de afrontamiento ante la muerte y género, entre nuestros resultados destaca que las mujeres tienden a utilizar un mayor repertorio de estrategias haciendo un uso combinado de los estilos de afrontamiento activo y evitativo, mientras que los hombres tienen una mayor capacidad de afrontamiento de la muerte, siendo la estrategia activa de Aceptación, la única que, en ellos, se asocia positivamente con la capacidad de afrontamiento de la muerte.

Por otro lado, también observamos similitudes; en ambos sexos el mayor uso de estilo de afrontamiento evitativo conlleva una menor capacidad de afrontamiento de la muerte, (aunque esta asociación es más intensa en el caso de los hombres).

La primera implicación a nivel de intervención en procesos de afrontamiento de la muerte, es que el estilo y las estrategias de afrontamiento que utiliza cada persona son áreas importantes a explorar. Por un lado, entre hombres y mujeres existen diferencias y similitudes, y por otro, como se observa, ser hombre o mujer no determina ni el estilo ni las estrategias de afrontamiento (Martin y Doka, 1996, 2000). Por tanto, no será tan útil clasificar hombres y mujeres en cuanto a los estilos de afrontamiento, sino realizar un análisis pormenorizado de los estilos y estrategias que cada uno utiliza. Por ejemplo, la aceptación en ambos sexos es una estrategia de afrontamiento destacable a trabajar para el afrontamiento de la muerte.

Además, estos resultados nos llevan a destacar la importancia de permitir a las personas utilizar estrategias evitativas combinadas con estrategias activas, no solamente en sus primeras fases del proceso de afrontamiento de muerte, como suele ser habitual, sino incluso en fases más avanzadas. Como hemos visto, en general en mujeres, el uso de estrategias de evitación no impide ni la aceptación ni el estilo de afrontamiento activo. 
Respecto a la asociación de otro tipo de variables con los distintos estilos de afrontamiento, como se ha visto previamente (Bermejo et al, 2012; López et al. 2003), las personas que profesan una religión y las que la practican, utilizan en mayor medida un estilo de afrontamiento activo. Sin embargo, en el marco de la atención sanitaria y de la psicoterapia, las variables relacionadas con la espiritualidad, como la religión, las prácticas de oración, meditación, etc., no siempre han estado presentes. Y quienes menos utilizan el estilo de afrontamiento activo son los auxiliares de enfermería y las personas que han sufrido pérdidas por enfermedad o por migración. Constatamos pues que, para un abordaje psicoterapéutico de calidad, han de considerarse múltiples variables, siendo el sexo una de ellas, sin hacer juicios deterministas en ese sentido.

No hay que perder de vista en la interpretación de los resultados el hecho de que los cuestionarios se pasasen a una muestra de personas que asistían a unas Jornadas de Duelo organizadas en un centro sociosanitario concreto. Con ello, podríamos estar seleccionando un perfil de personas con una cultura determinada respecto al afrontamiento de la muerte, pero por otro lado, es importante tener en cuenta que gracias a ello se ha podido seleccionar una muestra, en la que la mayor parte de las personas han vivido de cerca una experiencia de duelo.

En resumen, el presente estudio ofrece una nueva estructura más parsimoniosa de la escala COPE abreviada para población española y concluye sobre la importancia de tener en cuenta múltiples variables para el afrontamiento de la muerte, como la religión, los estudios, la carrera profesional y el tipo de pérdida, siendo el género una de ellas. Es destacable que las mujeres de nuestro estudio utilizan un mayor repertorio de estrategias de afrontamiento que, a largo plazo les puede dotar de una mayor capacidad de afrontamiento de la muerte, por lo que es importante permitirlo. Y, mientras que en mujeres no está reñido el uso combinado de ambos estilos de afrontamiento, activo y evitativo, en hombres el uso del estilo evitativo, parece estar contrapuesto a la aceptación, que se presenta como la estrategia más asociada a la capacidad de afrontamiento de la muerte en ambos sexos.

\section{Referencias Bibliográficas}

Abad, F. J., Olea, J., Ponsoda, V. y García, C. (2011). Medición en Ciencias Sociales y de la Salud. Madrid, España: Editorial Síntesis.

Bermejo, J. C., Magaña, M., Villacieros, M., Carabias, R. y Serrano, I. (2012). Estrategias de afrontamiento y resiliencia como factores mediadores de duelo complicado. Revista de Psicoterapia, 22(88), 85-95. Recuperado de: https://tienda.revistadepsicoterapia.com/revistas/aplicaciones-psicoterapeuticas.html

Bermejo, J. C. y Magaña, M. (2013). “Modelo Humanizar” de Intervención en Duelo. Santander, España: Sal Terrae.

Bonanno, G. A., Keltner, D., Holen, A. y Horowitz, M. J. (1995). When avoiding unpleasant emotions might not be such a bad thing: Verbal-autonomic response dissociation and midlife conjugal bereavement. Journal of Personality and Social Psychology, 69, 975-989.

Bugen, L. A. (1980). Coping: Effects of death education. Journal of Death and Dying, 81(11), 175-83. 
Cabanach, R. G., Fariña, F., Freire, C., González, P. y Ferradás, M. M. (2013). Diferencias en el afrontamiento del estrés en estudiantes universitarios hombres y mujeres. European Journal of Education and Psychology, 6(1), 19-32.

Carver, C. S. (1989). Assessing coping strategies: a theoretically based approach. Journal of personality and social psychology, 56(2), 267-283.

Carver, C. S. (1997). You want to measure coping but your protocol's too long: consider the Brief COPE. International Journal of Behavioral Medicine, 4, 92-100.

Corr, C. A., Nabe, C. M. y Corr, D. M. (2000). Death and dying, life and living (3rd ed.). Belmont, CA: Wadsworth/ Thomson Learning.

Elvira de la Morena, M. J. y Cruzado, J. A. (2013). Caregivers of patients with disorders of consciousness: coping and prolonged grief. Acta Neurologica Scandinavica, 127, 413-418. http://dx.doi.org/10.1111/ane.12061

Fernández-Abascal, E. G., Jiménez, P. y Martín, D. (2003). Emoción y Motivación. Madrid, España: Centro de Estudios Ramón Areces.

Galiana, L. (2015). Desenvolupament, Estudi i Ús d'Instruments de Mesura en Cures Palliatives: Aportacions teòric-pràctiques en malalts i professionals (Tesis Doctoral, Universidad de Valencia, Valencia, España). Recuperado de: https://www.educacion.gob.es/teseo/mostrarRef.do?ref=1129809

Galiana, L., Oliver, A., Sansó, N., Pades, A. y Benito, E. (2017). Validación confirmatoria de la Escala de Afrontamiento de la Muerte en profesionales de cuidados paliativos. Revista de Medicina Paliativa, 24(3), 126-135.

Hair, J., Anderson, R., Tatham, R. y Black, W. (1999). Análisis multivariante. Madrid, España: Prentice Hall.

Hu, L., y Bentler P. M. (1998). Fit indices in covariance structure modeling: Sensitivity to underparameterized model misspecification. Psychological Methods, 3, 424-453. https://doi.org/10.1037/1082-989X.3.4.424

Lazarus, R. S. y Folkman, S. (1984). Stress, appraisal and coping. Nueva York, NY: Springer.

López, J., Crespo, M., Arinero, M., Gómez, M. y Francisco, N. (2003). Initial analysis of psychometric properties of the brief-COPE in a sample of caregivers of older relatives. VII European conference on psychological assessment. Madrid, España: Universidad Complutense.

Martin, T. L. y Doka, K. J. (1996). Masculine grief. En K. L. Doka (Ed.), Living with grief after sudden loss (pp. 161-171). Bristol, PA: Taylor \& Francis.

Martin, T. L. y Doka, K. J. (2000). Men don't cry . . . women do. Transcending gender stereotypes of grief. Philadelphia, PA: Taylor \& Francis.

Martín, G., Lucas, B. y Pulido, R. (2011). Diferencias de género en el afrontamiento en la adolescencia. Brocas, $35,157-166$.

Martínez, J. P. (2015). Cómo se defiende el profesorado de secundaria del estrés: burnout y estrategias de afrontamiento. Journal of Work and Organizational Psychology, 31, 1-9.

Morales, F. M. y García, T. (2017). Efecto del entorno y rendimiento académico en las estrategias de afrontamiento infantil. Revista de estudios e investigación en psicología y educación, 14, 33-37.

Morán, C., Landero, R. y González, M. T. (2010). COPE-28: un análisis psicométrico de la versión en español del Brief COPE. Universitas Psychologica, 9(2), 543-552.

Neimeyer, R. A. (1997). Métodos de evaluación de la ansiedad ante la muerte. Madrid, España: Paidos.

Nolen-Hoeksema, S. y Larson, J. (1999). Coping with loss. Mahwah, NJ: Lawrence Erlbaum Associates.

Osterweis, M., Solomon, F. y Green, M. (1984). Bereavement: Reactions, consequences and care. Washington, DC: National Academy Press.

Perczek, R., Carver, C. S., Price, A. A. y Pozo-Kaderman, C. (2014). Coping, Mood, and Aspects of Personality in Spanish Translation and Evidence of Convergence with English Versions. Journal of personality assessment, 74(1), 63-87.

Riquelme, A. J., Rodríguez, M. C. y Buendía, J. (1993). Estrategias de afrontamiento y apoyo social en personas con estrés económico. Psicothema, 5(1), 83-89.

Rodríguez Marín, J. (1996). Psicología Social de la Salud. Madrid, España: Síntesis Psicología

Sadín, B. y Chorot, P. (2003). Cuestionario de Afrontamiento del Estrés (CAE): desarrollo y validación preliminar. Revista de psicopatología y psicología clínica, 8(1), 39-54.

Schmidt, J. (2007). Validación de la versión española de la escala Bugen de afrontamiento de la muerte y del perfil revisado de actitudes hacia la muerte. Estudio comparativo y transcultural. Puesta en marcha de un programa de intervención (Tesis doctoral no publicada, Universidad de Granada, Granada, España).

Selye, H. (1983). The Stress concept: past, present and future. En C. L. Cooper (ed.), Stress research: issues for the eighties (pp.1-20). Nueva York, NY: John Wiley \& Sons.

Staudacher, C. (1991). Men and grief. Oakland, CA: New Harbinger Publications. 
Stinson, K. M. y Lasker, J. N. (1992). Parents' grief following pregnancy loss: A comparison of mothers and fathers. Family Relations, 41, 218-224.

Villacieros, M., Serrano, I., Bermejo, J. C., Magaña, M. y Carabias, R. (2014). Social support and psychological well-being as possible predictors of complicated grief in a cross-section of people in mourning. Anales de Psicología, 30(3), 944-951. http://dx.doi.org/10.6018/analesps.30.3.154691.

Wartella, J. E., Auerbach, S. M. y Ward, K. R. (2009). Emotional distress, coping and adjustment in family members of neuroscience intensive care unit patients. Journal of Psychosomatic Research, 66, 503-509. http:// dx.doi.org/10.1016/j.jpsychores.2008.12.005 